\title{
Preface of the Special Issue "Pharmaceutical Crystals"
}

\author{
Etsuo Yonemochi ${ }^{1, *}$ (i) and Hidehiro Uekusa ${ }^{2, *(1)}$ \\ 1 Department of Physical Chemistry, School of Pharmacy and Pharmaceutical Sciences, Hoshi University, \\ 2-4-41 Ebara, Shinagawa-ku, Tokyo 142-8501, Japan \\ 2 Department of Chemistry, School of Sciences, Tokyo Institute of Technology, 2-12-1 Ookayama, Meguro-ku, \\ Tokyo 152-8551, Japan \\ * Correspondence: e-yonemochi@hoshi.ac.jp (E.Y.); uekusa@chem.titech.ac.jp (H.U.); \\ Tel.: +81-3-5498-5048 (E.Y.); +81-3-5734-3529 (H.U.)
}

Received: 1 February 2020; Accepted: 2 February 2020; Published: 5 February 2020

check for updates

Dear Colleagues,

We are delighted to deliver the "Pharmaceutical Crystals" Special Issue of Crystals.

The crystalline state is the most used and essential form of solid active pharmaceutical ingredients (APIs). The characterization of pharmaceutical crystals encompasses numerous scientific disciplines, and its center is crystal structure analysis, which reveals the molecular structure of important pharmaceutical compounds, This analysis also affords key structural information that relates to the broadly variable physicochemical properties of the APIs, such as solubility, stability, tablet ability, color, and hygroscopicity.

The Special Issue on "Pharmaceutical Crystals" aimed to publish novel molecular and crystal structures of pharmaceutical compounds, especially new crystal structures of APIs, including polymorphs and solvate crystals, as well as multi-component crystals of APIs such as co-crystals and salts. Although these pharmaceutical crystals have the same API, they may lead to different physicochemical properties depending on their unique structures.

Thus, this Special Issue demonstrates the importance of crystal structure information in many sectors of pharmaceutical science. Ten groups, both from industry and academia, contributed their latest studies that include morphology, spectroscopic, theoretical calculation, and thermal analysis with the crystallographic study. This wide variety of studies is the key to this Special Issue presenting current trends in the structure-property study of pharmaceutical crystals.

In this Special Issue, physicochemical properties and crystal structure are the focus, and a variety of properties were correlated to crystal structure. Solubility of a pharmaceutical crystal is one of the most exciting topics, and two groups contributed to this aspect. Ren et al. [1] studied the relationship between the solubility and crystal faces and crystal habits, providing a new idea on the mechanism of crystal habit modification and its impact on solubility. Co-crystal formation is known as one of the effective methods to improve solubility. Zhang et al. [2] found a novel co-crystal of the potent $\mathrm{H} 2$ receptor antagonist famotidine (FMT) with masonic acid, which was stable, and showed higher solubility than the intact crystalline phase. Another essential property of photostability was evaluated in carbamazepine polymorphs (forms I to III) and three co-crystals [3]. Yutani et al. fully utilized FT-IR, low-frequency Raman spectroscopy, and solid-state NMR to find that lower molecular mobility is the key to higher photostability. The chemical reactivity of pharmaceutical salt, nitrofurantoin-4-dimethylaminopyridine (NF-DMAP), was examined by Khan et al. [4] using DFT methods and spectroscopy to conclude that the API was chemically less reactive compared to the salt.

Dynamic phenomena such as dehydration phase transformation and solvent-mediated phase transformation are also an important aspect of pharmaceutical crystals because they relate to the stability of crystals. Dehydration behavior of ondansetron hydrochloride and hydrobromide was reported by Mizoguchi et al. [5] to elucidate the mechanism. They utilized a recently developed 
"Structure Determination from Powder Diffraction Data (SDPD)" technique to analyze the dehydrated crystal structures. Solvent-mediated polymorphic transformation of famoxadone from form II to form I was disclosed by $\mathrm{Du}$ et al. [6]. The transformation process was monitored by process analytical technologies and was found to be controlled by form I growth. It is interesting that hydrogen-bonding ability and dipolar polarizability affected the transformation.

How do crystals grow? Huang et al. successfully monitored the co-crystallization process in solution by Raman spectroscopy [7]. The authors found that suspension density and temperature both have an impact on the co-crystal formation. Besides the crystal growth, identification of the co-crystal composition is the critical step of any further analysis. An et al. successfully utilized the melting diagrams for adefovir dipivoxil and dicarboxylic acids [8]. This method is powerful in assessing the co-crystal composition in solid-state crystallization.

Molecular docking is an emerging topic for pharmaceutical crystal study. Ivashchenko et al. reported the crystal structure of a new biologically active molecule, which was also investigated as a new inhibitor of hepatitis B in a molecular docking study [9]. This substance has in vitro nanomolar inhibitory activity against the hepatitis B virus (HBV). Another docking study was reported by Al-Wabli et al. [10], where a newly synthesized compound was crystallographically characterized. Furthermore, the structure was analyzed using molecular docking studies and Hirshfeld surface analysis. The in vitro antifungal potential of the compound was examined against four different fungal strains.

In conclusion, this Special Issue presents a wide range of recent studies about pharmaceutical crystals and provides valuable information for future studies in the related fields. The guest editors hope the readers enjoy this beneficial Special Issue of "Pharmaceutical Crystals".

Prof. Etsuo Yonemochi

Prof. Hidehiro Uekusa

Guest Editors

\section{References}

1. Ren, Y.; Shen, J.; Yu, K.; Phan, C.U.; Chen, G.; Liu, J.; Hu, X.; Feng, J. Impact of Crystal Habit on Solubility of Ticagrelor. Crystals 2019, 9, 556. [CrossRef]

2. Zhang, Y.; Yang, Z.; Zhang, S.; Zhou, X. Synthesis, Crystal Structure, and Solubility Analysis of a Famotidine Cocrystal. Crystals 2019, 9, 360. [CrossRef]

3. Yutani, R.; Haku, R.; Teraoka, R.; Tode, C.; Koide, T.; Kitagawa, S.; Sakane, T.; Fukami, T. Comparative Evaluation of the Photostability of Carbamazepine Polymorphs and Co-crystals. Crystals 2019, 9, 553. [CrossRef]

4. Khan, E.; Shukla, A.; Srivastava, K.; Gangopadhyay, D.; Assi, K.H.; Tandon, P.; Vangala, V.R. Structural and Reactivity Analyses of Nitrofurantoin-4-dimethylaminopyridine Salt Using Spectroscopic and Density Functional Theory Calculations. Crystals 2019, 9, 413. [CrossRef]

5. Mizoguchi, R.; Uekusa, H. Elucidation of the Crystal Structures and Dehydration Behaviors of Ondansetron Salts. Crystals 2019, 9, 180. [CrossRef]

6. Du, D.; Ren, G.-B.; Qi, M.-H.; Li, Z.; Xu, X.-Y. Solvent-Mediated Polymorphic Transformation of Famoxadone from Form II to Form I in Several Mixed Solvent Systems. Crystals 2019, 9, 161. [CrossRef]

7. Huang, Y.; Zhou, L.; Yang, W.; Li, Y.; Yang, Y.; Zhang, Z.; Wang, C.; Zhang, X.; Yin, Q. Preparation of Theophylline-Benzoic Acid Cocrystal and On-Line Monitoring of Co-crystallization Process in Solution by Raman Spectroscopy. Crystals 2019, 9, 329. [CrossRef]

8. An, H.; Choi, I.; Kim, I.W. Melting Diagrams of Adefovir Dipivoxil and Dicarboxylic Acids: An Approach to Assess Co-crystal Compositions. Crystals 2019, 9, 70. [CrossRef]

9. Ivashchenko, A.V.; Mitkin, O.D.; Kravchenko, D.V.; Kuznetsova, I.V.; Kovalenko, S.M.; Bunyatyan, N.D.; Langer, T. Synthesis, X-Ray Crystal Structure, Hirshfeld Surface Analysis, and Molecular Docking Study of Novel Hepatitis B (HBV) Inhibitor: 8-Fluoro-5-(4-fluorobenzyl)-3-(2-methoxybenzyl)-3,5-dihydro-4H-pyrimido[5,4-b]indol-4-one. Crystals 2019, 9, 379. [CrossRef] 
10. Al-Wabli, R.I.; Al-Ghamdi, A.R.; Aswathy, S.A.V.; Ghabbour, H.A.; Al-Agamy, M.H.; Hubert Joe, I.; Attia, M.I. (2E)-2-[1-(1,3-Benzodioxol-5-yl)-3-(1H-imidazol-1-yl)propylidene]-N-(2-chlorophenyl)hydrazine carboxamide: Synthesis, X-ray Structure, Hirshfeld Surface Analysis, DFT Calculations, Molecular Docking and Antifungal Profile. Crystals 2019, 9, 82. [CrossRef] 